\title{
ALLELOPATHIC POTENTIAL OF CAPSICUM ANNUUM L. AND CORIANDRUM SATIVUM L. ON GROWTH OF BEAN CROP
}

\author{
Muhammad Zafar IQBAL ${ }^{1}$, Lubna AHMED ${ }^{1}$, Muhammad SHAFIQ ${ }^{1 *}$ \\ ${ }^{1}$ Department of Botany, University of Karachi, Karachi - 75270, Pakistan \\ ${ }^{*}$ Corresponding author. E-mail: shafiqeco@yahoo.com
}

\begin{abstract}
Allelochemical are natural compounds which effects the growth of surrounding plants. The treatment of aqueous powder extracts of Capsicum annuum L. and Coriandrum sativum L. at $1 \%$ showed significant decreased in shoot, root and seedling height, leaves growth and biomass of mung bean (Vigna radiata). The inhibitory effect on growth performance was directly related with the increasing concentration of powder extracts of coriander. The seedlings of both legume bean were tested for the determination of toxicity and tolerance to red chilli and coriander powder extract treatment at $0,1,2$, 3, 4 and 5\%. The seedlings of mung bean and cowpea showed lowest percentage of tolerance indices to coriander and red chilli powder extract treatment at 5\%. However, on comparison, the seedlings of cowpea showed more tolerance to coriander and red chili powder extract than mung bean.
\end{abstract}

Keywords: coriander, phytotoxicity, plant extracts, red chili, root growth, seedling growth, tolerance indices.

\section{Introduction}

The discharge of phytochemical substances from one plant species altered the growth performances of surrounding plants. Many studies have shown that the release of toxic substances by a plant decreased or promote growth growth of neighboring plants [CHOU \& LEE, 1991; KIL \& YUN, 1992; REINHARDT \& al. 1993; ALLOLI \& NARAYANREDDY, 2000; DAIZY \& al. 2001; FERGUSON \& RATHINASABPATHI, 2003; OUSSAMA, 2003; DONGRE \& YADAV, 2005; MISHRA \& al. 2014] and termed as allelopathy. The impact of leaf leachates of some woody plant species on agriculture and some desertt plants were reported [MELKANIA, 1984; HEGAZY \& al. 1990; PURI \& KHARA, 1991]. Seed regeneration failures of Pinus silvestris L. by Empetrum hermaphroditum Hagerup occurred due to allelopathic interference [ZACKRISSON \& NILSSON, 1992].

Allelopathy helps in ecosystems management [ABBASSI \& al. 2013] and some work in earlies 1970 was initiated in Pakistan [CHEEMA \& al. 2013]. These chemicals products can serve for weed control [BAGHERI \& al. 2013; BOJOVIĆ \& JAKOVLJEVIĆ, 2015]. The release of allelopathic compounds influence positively and negatively on the growth of plants. Capsicum annuum L. belongs to Solanaceae family. Coriandrum sativum L. (Apiaceae) is popular medicinal plant and seeds are source of iron, copper, calcium, magnesium and zinc. Both spices production have great economic demands and used in cooking on daily basis in Pakistan and worldwide.

The purpose of the studies was to assess the toxic potential of red chili and coriander aqueous powders extract on the growth of two different bean crops namely, cowpea and mung bean because of economic importance of both leguminous crops are cultivating in the larger agricultural area of the Pakistan. 


\section{Material and methods}

The experimental site is located in the Department of Botany at the Karachi University Campus and seedling growth experiment was carried out in pots in green house. $1 \%$ solution of red chilli or coriander powder prepared by weighing one $\mathrm{g}$ of spice powder then dissolve in $99 \mathrm{ml}$ of distilled water to make up the volume up to $100 \mathrm{ml}$ and subsequent concentrations $1 \%, 2 \%, 3 \%, 4 \%$ and $5 \%$ were prepared respectively. The fruit of red chilli and seeds of coriander powder was kept in boiling so that convert into solution completely. The certified seeds of cowpea and mung bean were purchased from market and imbibed in water for an hour to break seed dormancy. The beans seeds surface was sterilized with $1 \mathrm{~N}$ bleach solution for three minutes and rinsed by distilled water to remove any type of fungal contamination. The plastic pots were $7.3 \mathrm{~cm}$ in diameter and $9.6 \mathrm{~cm}$ in depth and were filled with ratio of one-part manure and three parts garden loam. Dig $1 \mathrm{~cm}$ holes of soil from above the surface of pots, at least 5 holes and buried the 2-2 seeds of one type of plant into pot. The pots were water daily and under the influence of sunlight, seeds were able to germinate uniformly in two weeks. One seedling was grown in each pot and ten ml of powder extract of Capsicum annuum L. and Coriandrum sativum L. were provided into the respective pots. The experiment was completely randomized. The pots were reshuffled weekly to avoid light, shade or any other climatic factors. There were five replicates of each treatment. The seedlings were uprooted from the pots after five weeks and washed with tap water. The root, shoot and seedling length and numbers of leaves were recorded. The removed seedlings were kept in oven at $80^{\circ} \mathrm{C}$ for 24 hours for the determination of dry weight. Root, leaf, shoot and total plant fresh and dry weight were recorded. Root / shoot ratio, leaf weight, specific leaf area and leaf area ratio were determined according to REHMAN \& IQBAL (2009).

The obtained data was statistically analyzed by ANOVA and DMRT (Duncan Multiple Range Test) ( $\mathrm{p}$ < 0.05) using software packages SPSS version 14.0 on personal computer.

\section{Results}

The treatment of different concentrations of red chilli and coriander aqueous powder extract showed variable effects on growth performance of cowpea and mung bean (Table 14). Red chilli extract treatment at $4 \%$ significantly $(\mathrm{p}<0.05)$ decreased root, shoot, seedling height, leaf area and total fresh weight of cow pea. The significant decline in leaf weight ratio of cow pea was found at $3 \%$ red chilli extract treatment. The treatment of red chilli powder extract at $3 \%$ was brought a significant decrease in shoot growth, number of leaves and leaves dry weight of mung bean. The treatment of red chilli at all concentration showed nonsignificant effect on root, shoot dry weight and specific leaf area of cow pea, whereas, root fresh weight and root shoot ratio of mung bean. Shoot, root, seedling length, number of leaves and leaf size of cow pea was highly decreased at $5 \%$ coriander aqueous powder extract treatment. Coriander extract treatment at $1 \%$ treatment produced significantly lower number of leaves in mung bean. The coriander extract treatment at $5 \%$ significantly affected root, shoot and total plant dry weight of mung bean. Leaf weight ratio, of mung bean greatly affected by $5 \%$ of coriander powder extract treatment. 
Muhammad Zafar IQBAL \& al.

Table 1. Growth of cowpea in various concentrations of red chilli powder extract.

\begin{tabular}{|c|c|c|c|c|c|c|}
\hline \multirow{2}{*}{$\begin{array}{l}\text { Seedling growth } \\
\text { characteristic }\end{array}$} & \multicolumn{6}{|c|}{ Treatments aqueous powder extract concentration (\%) } \\
\hline & $\mathbf{0}$ & 1 & 2 & 3 & 4 & 5 \\
\hline Shoot length $(\mathrm{cm})$ & $\begin{array}{l}69.31 \mathrm{f} \\
\pm 0.070\end{array}$ & $\begin{array}{l}68.86 \mathrm{e} \\
\pm 0.017\end{array}$ & $\begin{array}{l}66.67 \mathrm{~d} \\
\pm 0.017\end{array}$ & $\begin{array}{l}66.56 \text { c } \\
\pm 0.015\end{array}$ & $\begin{array}{l}60.32 \mathrm{~b} \\
\pm 0.006\end{array}$ & $\begin{array}{l}60.00 \mathrm{a} \\
\pm 0.001\end{array}$ \\
\hline Root length (cm) & $\begin{array}{l}12.75 \mathrm{f} \\
\pm 0.020\end{array}$ & $\begin{array}{l}12.05 \mathrm{e} \\
\pm 0.020\end{array}$ & $\begin{array}{l}10.80 \mathrm{~d} \\
\pm 0.011\end{array}$ & $\begin{array}{c}9.87 \text { c } \\
\pm 0.004\end{array}$ & $\begin{array}{l}9.50 \mathrm{~b} \\
\pm 0.040\end{array}$ & $\begin{array}{c}8.27 \mathrm{a} \\
\pm 0.016\end{array}$ \\
\hline Seedling length $(\mathrm{cm})$ & $\begin{array}{l}82.05 \mathrm{f} \\
\pm 0.054\end{array}$ & $\begin{array}{l}80.91 \mathrm{e} \\
\pm 0.006\end{array}$ & $\begin{array}{l}77.47 \mathrm{~d} \\
\pm 0.099\end{array}$ & $\begin{array}{l}76.43 \text { c } \\
\pm 0.018\end{array}$ & $\begin{array}{l}69.82 \mathrm{~b} \\
\pm 0.044\end{array}$ & $\begin{array}{l}68.27 \mathrm{a} \\
\pm 0.009\end{array}$ \\
\hline Number of leaves & $\begin{array}{c}15.00 \text { ae } \\
\pm 0.00\end{array}$ & $\begin{array}{l}13.66 \mathrm{~d} \\
\pm 0.710\end{array}$ & $\begin{array}{l}12.33 \text { c } \\
\pm 0.062\end{array}$ & $\begin{array}{l}12.00 \mathrm{bc} \\
\pm 0.001\end{array}$ & $\begin{array}{l}11.00 \mathrm{ab} \\
\pm 0.010\end{array}$ & $\begin{array}{l}10.33 \mathrm{a} \\
\pm 0.023\end{array}$ \\
\hline Leaf area $\left(\mathrm{cm}^{2}\right)$ & $\begin{array}{l}48.65 \mathrm{~d} \\
\pm 0.037\end{array}$ & $\begin{array}{l}42.91 \mathrm{~d} \\
\pm 0.007\end{array}$ & $\begin{array}{l}38.00 \text { c } \\
\pm 0.408\end{array}$ & $\begin{array}{l}35.33 \mathrm{~b} \\
\pm 0.082\end{array}$ & $\begin{array}{l}35.58 \mathrm{~b} \\
\pm 0.129\end{array}$ & $\begin{array}{l}30.85 \mathrm{a} \\
\pm 0.042\end{array}$ \\
\hline Root fresh weight (g) & $\begin{array}{l}0.880 \mathrm{~d} \\
\pm 0.020\end{array}$ & $\begin{array}{l}0.860 \mathrm{~d} \\
\pm 0.006\end{array}$ & $\begin{array}{l}0.800 \text { c } \\
\pm 0.004\end{array}$ & $\begin{array}{l}0.640 \mathrm{~b} \\
\pm 0.004\end{array}$ & $\begin{array}{l}0.620 \mathrm{~b} \\
\pm 0.018\end{array}$ & $\begin{array}{l}0.440 \mathrm{a} \\
\pm 0.008\end{array}$ \\
\hline Shoot fresh weight (g) & $\begin{array}{c}1.72 \mathrm{e} \\
\pm 0.006 \\
\end{array}$ & $\begin{array}{r}1.42 \mathrm{~d} \\
\pm 0.006 \\
\end{array}$ & $\begin{array}{c}1.34 \mathrm{c} \\
\pm 0.004 \\
\end{array}$ & $\begin{array}{c}1.33 \mathrm{c} \\
\pm 0.004 \\
\end{array}$ & $\begin{array}{r}1.02 \mathrm{~b} \\
\pm 0.020 \\
\end{array}$ & $\begin{array}{l}0.850 \mathrm{a} \\
\pm 0.012 \\
\end{array}$ \\
\hline Leaves fresh weight (g) & $\begin{array}{c}1.15 \mathrm{~d} \\
\pm 0.009\end{array}$ & $\begin{array}{c}1.01 \\
\pm 0.230\end{array}$ & $\begin{array}{l}0.870 \text { bc } \\
\pm 0.006\end{array}$ & $\begin{array}{c}0.640 \mathrm{ab} \\
\pm 0.012\end{array}$ & $\begin{array}{l}0.620 \mathrm{ab} \\
\pm 0.005\end{array}$ & $\begin{array}{c}0.440 \mathrm{a} \\
\pm 0.008\end{array}$ \\
\hline Total plant fresh weight (g) & $\begin{array}{c}3.30 \mathrm{~d} \\
\pm 0.106\end{array}$ & $\begin{array}{l}2.290 \mathrm{c} \\
\pm 0.206\end{array}$ & $\begin{array}{l}2.350 \mathrm{c} \\
\pm 0.16\end{array}$ & $\begin{array}{l}2.11 \text { bc } \\
\pm 0.012\end{array}$ & $\begin{array}{c}1.890 \mathrm{ab} \\
\pm 0.092 \\
\end{array}$ & $\begin{array}{c}1.760 \mathrm{ab} \\
\pm 0.082\end{array}$ \\
\hline Root dry weight (g) & $\begin{array}{l}0.180 \mathrm{a} \\
\pm 0.430\end{array}$ & $\begin{array}{l}0.190 \mathrm{a} \\
\pm 0.025\end{array}$ & $\begin{array}{l}0.310 \mathrm{a} \\
\pm 0.004\end{array}$ & $\begin{array}{l}0.170 \mathrm{a} \\
\pm 0.004\end{array}$ & $\begin{array}{l}0.120 \mathrm{a} \\
\pm 0.005\end{array}$ & $\begin{array}{l}0.100 \mathrm{a} \\
\pm 0.198\end{array}$ \\
\hline Shoot dry weight (g) & $\begin{array}{l}0.850 \mathrm{e} \\
\pm 0.012\end{array}$ & $\begin{array}{l}0.720 \mathrm{~d} \\
\pm 0.004\end{array}$ & $\begin{array}{l}0.660 \text { с } \\
\pm 0.013\end{array}$ & $\begin{array}{l}0.680 \mathrm{c} \\
\pm 0.017\end{array}$ & $\begin{array}{l}0.520 b \\
\pm 0.004\end{array}$ & $\begin{array}{l}0.310 \mathrm{a} \\
\pm 0.004\end{array}$ \\
\hline Leaves dry weight (g) & $\begin{array}{l}0.280 \mathrm{a} \\
\pm 0.008\end{array}$ & $\begin{array}{r}0.260 \mathrm{a} \\
\pm 0.006\end{array}$ & $\begin{array}{l}0.250 \mathrm{a} \\
\pm 0.011\end{array}$ & $\begin{array}{l}0.240 \mathrm{a} \\
\pm 0.008\end{array}$ & $\begin{array}{l}0.185 \mathrm{a} \\
\pm 0.005\end{array}$ & $\begin{array}{l}0.100 \mathrm{a} \\
\pm 0.220\end{array}$ \\
\hline Total plant dry weight (g) & $\begin{array}{l}2.180 b \\
\pm 0.428 \\
\end{array}$ & $\begin{array}{c}2.020 \mathrm{ab} \\
\pm 0.220 \\
\end{array}$ & $\begin{array}{l}1.510 \mathrm{a} \\
\pm 0.070\end{array}$ & $\begin{array}{l}1.210 \mathrm{a} \\
\pm 0.039\end{array}$ & $\begin{array}{r}0.980 \mathrm{a} \\
\pm 0.041\end{array}$ & $\begin{array}{l}0.780 \mathrm{a} \\
\pm 0.020\end{array}$ \\
\hline Root / Shoot ratio & $\begin{array}{r}0.297 \mathrm{~b} \\
\pm 0.037 \\
\end{array}$ & $\begin{array}{r}0246 \text { a } \\
\pm 0.032 \\
\end{array}$ & $\begin{array}{r}0.350 \mathrm{a} \\
\pm 0.002 \\
\end{array}$ & $\begin{array}{r}0.310 \mathrm{a} \\
\pm 0.036 \\
\end{array}$ & $\begin{array}{c}0.316 \mathrm{ab} \\
\pm 0.032 \\
\end{array}$ & $\begin{array}{r}0.320 \mathrm{a} \\
\pm 0.063 \\
\end{array}$ \\
\hline Leaf weight ratio & $\begin{array}{c}0.450 \mathrm{ab} \\
\pm 0.007\end{array}$ & $\begin{array}{c}0.280 \mathrm{ab} \\
\pm 0.015\end{array}$ & $\begin{array}{r}0.220 \mathrm{a} \\
\pm 0.004 \\
\end{array}$ & $\begin{array}{l}0.230 \mathrm{a} \\
\pm 0.006\end{array}$ & $\begin{array}{l}0.310 b \\
\pm 0.011\end{array}$ & $\begin{array}{l}0.340 \mathrm{~b} \\
\pm 0.005\end{array}$ \\
\hline Specific leaf area $\left(\mathrm{cm}^{2} \mathrm{~g}^{-1}\right)$ & $\begin{array}{c}23.260 \mathrm{a} \\
\pm 0.004 \\
\end{array}$ & $\begin{array}{c}38.930 \mathrm{a} \\
\pm 0.122 \\
\end{array}$ & $\begin{array}{c}43.270 \mathrm{a} \\
\pm 0.148 \\
\end{array}$ & $\begin{array}{c}53.190 \mathrm{a} \\
\pm 0.169 \\
\end{array}$ & $\begin{array}{c}37.540 \mathrm{a} \\
\pm 0.104 \\
\end{array}$ & $\begin{array}{c}47.160 \mathrm{a} \\
\pm 0.242 \\
\end{array}$ \\
\hline Leaf area ratio $\left(\mathrm{cm}^{2} \mathrm{~g}^{-1}\right)$ & $\begin{array}{c}10.450 \mathrm{c} \\
\pm 0.107\end{array}$ & $\begin{array}{c}10.990 \text { c } \\
\pm 0.330\end{array}$ & $\begin{array}{l}9.910 \mathrm{a} \\
\pm 0.042\end{array}$ & $\begin{array}{c}12.620 b \\
\pm 0.036\end{array}$ & $\begin{array}{c}11.800 \mathrm{~d} \\
\pm 0.057\end{array}$ & $\begin{array}{c}11.550 \mathrm{e} \pm \\
0.102\end{array}$ \\
\hline
\end{tabular}


ALLELOPATHIC POTENTIAL OF CAPSICUM ANNUUM L. AND CORIANDRUM SATIVUM L. ON...

Table 2. Growth of mung bean in various concentrations of red chilli powder extract.

\begin{tabular}{|c|c|c|c|c|c|c|}
\hline \multirow{2}{*}{$\begin{array}{l}\text { Seedling growth } \\
\text { characteristic }\end{array}$} & \multicolumn{6}{|c|}{ Treatments aqueous powder extract concentration (\%) } \\
\hline & $\mathbf{0}$ & 1 & 2 & 3 & 4 & 5 \\
\hline Shoot length $(\mathrm{cm})$ & $\begin{array}{l}48.210 \mathrm{a} \\
\pm 0.048\end{array}$ & $\begin{array}{l}44.130 \mathrm{e} \\
\pm 0.041\end{array}$ & $\begin{array}{l}43.00 \mathrm{~d} \\
\pm 0.025\end{array}$ & $\begin{array}{l}42.810 \mathrm{c} \\
\pm 0.020\end{array}$ & $\begin{array}{c}42.330 \mathrm{~b} \\
\pm 0.213\end{array}$ & $\begin{array}{c}40.230 \mathrm{~b} \\
\pm 0.052\end{array}$ \\
\hline Root length (cm) & $\begin{array}{l}12.43 \mathrm{a} \\
\pm 0.094\end{array}$ & $\begin{array}{l}10.96 \mathrm{e} \\
\pm 0.251\end{array}$ & $\begin{array}{l}11.370 \mathrm{c} \\
\pm 0.094\end{array}$ & $\begin{array}{l}11.000 \mathrm{~d} \\
\pm 0.001\end{array}$ & $\begin{array}{l}8.060 \mathrm{~d} \\
\pm 0.043\end{array}$ & $\begin{array}{l}6.900 \mathrm{~b} \\
\pm 0.231\end{array}$ \\
\hline Seedling length (cm) & $\begin{array}{l}60.640 \mathrm{~b} \\
\pm 0.140\end{array}$ & $\begin{array}{c}55.090 \mathrm{a} \\
\pm 0.029\end{array}$ & $\begin{array}{c}54.370 \mathrm{~b} \\
\pm 0.071\end{array}$ & $\begin{array}{l}53.810 \mathrm{~b} \\
\pm 11.767\end{array}$ & $\begin{array}{c}50.390 \mathrm{~b} \\
\pm 0.172\end{array}$ & $\begin{array}{l}47.130 \mathrm{~b} \\
\pm 0.273\end{array}$ \\
\hline Number of leaves & $\begin{array}{l}10.00 \mathrm{a} \\
\pm 0.001\end{array}$ & $\begin{array}{l}8.660 \mathrm{c} \\
\pm 0.249\end{array}$ & $\begin{array}{l}8.000 \mathrm{~b} \\
\pm 0.002\end{array}$ & $\begin{array}{c}7.870 \mathrm{ab} \\
\pm 0.462\end{array}$ & $\begin{array}{l}7.510 \mathrm{a} \\
\pm 0.427\end{array}$ & $\begin{array}{l}7.120 \mathrm{a} \\
\pm 0.241\end{array}$ \\
\hline Leaf area $\left(\mathrm{cm}^{2}\right)$ & $\begin{array}{c}22.800 \mathrm{a} \\
\pm 0.218\end{array}$ & $\begin{array}{c}21.450 \mathrm{e} \\
\pm 0.114\end{array}$ & $\begin{array}{c}20.450 \mathrm{~d} \\
\pm 0.085\end{array}$ & $\begin{array}{c}20.300 \mathrm{c} \\
\pm 0.091\end{array}$ & $\begin{array}{l}18.000 \mathrm{c} \\
\pm 0.001\end{array}$ & $\begin{array}{l}17.000 \mathrm{~b} \\
\pm 0.002\end{array}$ \\
\hline Root fresh weight (g) & $\begin{array}{l}0.390 \mathrm{a} \\
\pm 0.009\end{array}$ & $\begin{array}{l}0.360 \mathrm{a} \\
\pm 0.004\end{array}$ & $\begin{array}{l}0.230 \mathrm{a} \\
\pm 0.013\end{array}$ & $\begin{array}{l}0.210 \mathrm{a} \\
\pm 0.006\end{array}$ & $\begin{array}{l}0.100 \mathrm{a} \\
\pm 0.223\end{array}$ & $\begin{array}{l}0.090 \mathrm{a} \\
\pm 0.004\end{array}$ \\
\hline Shoot fresh weight (g) & $\begin{array}{l}1.630 \mathrm{a} \\
\pm 0.165\end{array}$ & $\begin{array}{l}1.410 \mathrm{e} \\
\pm 0.006\end{array}$ & $\begin{array}{l}1.400 \mathrm{~d} \\
\pm 0.005\end{array}$ & $\begin{array}{l}1.210 \mathrm{~d} \\
\pm 0.013\end{array}$ & $\begin{array}{l}1.150 \mathrm{c} \\
\pm 0.004\end{array}$ & $\begin{array}{l}1.010 \mathrm{~b} \\
\pm 0.006\end{array}$ \\
\hline Leaves fresh weight (g) & $\begin{array}{l}1.280 \mathrm{~b} \\
\pm 0.021\end{array}$ & $\begin{array}{l}0.520 \mathrm{e} \\
\pm 0.006\end{array}$ & $\begin{array}{l}0.720 \mathrm{a} \\
\pm 0.002\end{array}$ & $\begin{array}{l}0.690 \mathrm{~d} \\
\pm 0.004\end{array}$ & $\begin{array}{l}0.640 \mathrm{c} \\
\pm 0.004\end{array}$ & $\begin{array}{l}0.620 \mathrm{~b} \\
\pm 0.005\end{array}$ \\
\hline Total plant fresh weight (g) & $\begin{array}{r}3.300 \mathrm{a} \\
\pm 0.019\end{array}$ & $\begin{array}{l}2.290 \mathrm{c} \\
\pm 0.011\end{array}$ & $\begin{array}{c}2.350 \mathrm{~b} \\
\pm 0.11\end{array}$ & $\begin{array}{l}2.110 \mathrm{~b} \\
\pm 0.017\end{array}$ & $\begin{array}{l}1.890 \mathrm{~b} \\
\pm 0.229\end{array}$ & $\begin{array}{l}1.720 \mathrm{ab} \\
\pm 0.006\end{array}$ \\
\hline Root dry weight (g) & $\begin{array}{l}0.170 \mathrm{a} \\
\pm 0.004\end{array}$ & $\begin{array}{c}0.150 \text { bc } \\
\pm 0.004\end{array}$ & $\begin{array}{l}0.190 \mathrm{~b} \\
\pm 0.004 \\
\end{array}$ & $\begin{array}{l}0.160 \mathrm{c} \\
\pm 0.004 \\
\end{array}$ & $\begin{array}{l}0.040 \mathrm{~b} \\
\pm 0.006\end{array}$ & $\begin{array}{l}0.020 \mathrm{a} \\
\pm 0.017 \\
\end{array}$ \\
\hline Shoot dry weight (g) & $\begin{array}{r}1.030 \mathrm{a} \\
\pm 0.004 \\
\end{array}$ & $\begin{array}{r}1.010 \mathrm{e} \\
\pm 0.006 \\
\end{array}$ & $\begin{array}{l}0.640 \mathrm{f} \\
\pm 0.004 \\
\end{array}$ & $\begin{array}{l}0.410 \mathrm{~d} \\
\pm 0.004 \\
\end{array}$ & $\begin{array}{r}0.350 \mathrm{c} \\
\pm 0.008 \\
\end{array}$ & $\begin{array}{l}0.210 \mathrm{~b} \\
\pm 0.002 \\
\end{array}$ \\
\hline Leaves dry weight (g) & $\begin{array}{r}0.980 \mathrm{a} \\
\pm 0.004\end{array}$ & $\begin{array}{l}0.860 \mathrm{f} \\
\pm 0.004\end{array}$ & $\begin{array}{l}0.680 \mathrm{e} \\
\pm 0.004\end{array}$ & $\begin{array}{l}0.590 \mathrm{c} \\
\pm 0.008\end{array}$ & $\begin{array}{l}0.550 \mathrm{~b} \\
\pm 0.004\end{array}$ & $\begin{array}{l}0.780 \mathrm{~b} \\
\pm 0.220\end{array}$ \\
\hline Total plant dry weight (g) & $\begin{array}{l}2.180 \mathrm{~b} \\
\pm 0.428\end{array}$ & $\begin{array}{c}2.020 \mathrm{ab} \\
\pm 0.220\end{array}$ & $\begin{array}{l}1.510 \mathrm{a} \\
\pm 0.070\end{array}$ & $\begin{array}{r}1.210 \mathrm{a} \\
\pm 0.039\end{array}$ & $\begin{array}{r}0.980 \mathrm{a} \\
\pm 0.041\end{array}$ & $\begin{array}{l}0.780 \mathrm{a} \\
\pm 0.020\end{array}$ \\
\hline Root / Shoot ratio & $\begin{array}{r}0.297 \mathrm{a} \\
\pm 0.004 \\
\end{array}$ & $\begin{array}{r}0.291 \mathrm{a} \\
\pm 0.043 \\
\end{array}$ & $\begin{array}{l}0.304 \mathrm{a} \\
\pm 0.002 \\
\end{array}$ & $\begin{array}{r}0.296 \mathrm{a} \\
\pm 0.020 \\
\end{array}$ & $\begin{array}{r}0.216 \mathrm{a} \\
\pm 0.028 \\
\end{array}$ & $\begin{array}{l}0.192 \mathrm{a} \\
\pm 0.006 \\
\end{array}$ \\
\hline Leaf weight ratio & $\begin{array}{r}0.450 \mathrm{a} \\
\pm 0.001 \\
\end{array}$ & $\begin{array}{l}0.425 \mathrm{a} \\
\pm 0.011 \\
\end{array}$ & $\begin{array}{r}0.450 \mathrm{a} \\
\pm 0.001 \\
\end{array}$ & $\begin{array}{c}0.450 \mathrm{ab} \\
\pm 0.004 \\
\end{array}$ & $\begin{array}{r}0.602 \mathrm{a} \\
\pm 0.051 \\
\end{array}$ & $\begin{array}{l}0.705 \mathrm{~b} \\
\pm 0.014 \\
\end{array}$ \\
\hline Specific leaf area $\left(\mathrm{cm}^{2} \mathrm{~g}^{-1}\right)$ & $\begin{array}{c}23.260 \mathrm{a} \\
\pm 0.242\end{array}$ & $\begin{array}{c}24.900 \mathrm{~b} \\
\pm 0.095\end{array}$ & $\begin{array}{c}30.070 \mathrm{c} \\
\pm 0.052\end{array}$ & $\begin{array}{c}31.710 \mathrm{~d} \\
\pm 0.100 \\
\end{array}$ & $\begin{array}{c}30.500 \mathrm{c} \\
\pm 0.097\end{array}$ & $\begin{array}{c}30.900 \mathrm{c} \\
\pm 0.176\end{array}$ \\
\hline Leaf area ratio $\left(\mathrm{cm}^{2} \mathrm{~g}^{-1}\right)$ & $\begin{array}{l}10.450 \mathrm{a} \\
\pm 0.069\end{array}$ & $\begin{array}{c}10.610 \mathrm{a} \\
\pm 0.112\end{array}$ & $\begin{array}{c}13.540 \mathrm{~b} \\
\pm 0.070\end{array}$ & $\begin{array}{c}16.770 \mathrm{c} \\
\pm 0.155\end{array}$ & $\begin{array}{c}18.360 \mathrm{~d} \\
\pm 0.184\end{array}$ & $\begin{array}{c}21.790 \mathrm{e} \pm \\
0.154\end{array}$ \\
\hline
\end{tabular}


Muhammad Zafar IQBAL \& al.

Table 3. Growth of cowpea in various concentrations of coriander powder extract.

\begin{tabular}{|c|c|c|c|c|c|c|}
\hline \multirow{2}{*}{$\begin{array}{l}\text { Seedling growth } \\
\text { characteristic }\end{array}$} & \multicolumn{6}{|c|}{ Treatments aqueous powder extract concentration (\%) } \\
\hline & $\mathbf{0}$ & 1 & 2 & 3 & 4 & 5 \\
\hline Shoot length $(\mathrm{cm})$ & $\begin{array}{l}69.30 \mathrm{f} \\
\pm 0.040\end{array}$ & $\begin{array}{l}65.87 \mathrm{~d} \\
\pm 0.067 \\
\end{array}$ & $\begin{array}{l}64.56 \mathrm{c} \\
\pm 0.157 \\
\end{array}$ & $\begin{array}{l}68.92 \mathrm{e} \\
\pm 0.195 \\
\end{array}$ & $\begin{array}{l}62.18 \mathrm{~b} \\
\pm 0.008 \\
\end{array}$ & $\begin{array}{l}57.00 \mathrm{a} \\
\pm 0.278\end{array}$ \\
\hline Root length (cm) & $\begin{array}{l}12.75 \mathrm{c} \\
\pm 0.016 \\
\end{array}$ & $\begin{array}{l}10.80 \mathrm{~b} \\
\pm 0.040\end{array}$ & $\begin{array}{l}15.05 \mathrm{e} \\
\pm 0.012 \\
\end{array}$ & $\begin{array}{l}12.80 \mathrm{c} \\
\pm 0.173\end{array}$ & $\begin{array}{l}13.65 \mathrm{~d} \\
\pm 0.167\end{array}$ & $\begin{array}{c}8.60 \mathrm{a} \\
\pm 0.040\end{array}$ \\
\hline Seedling length (cm) & $\begin{array}{l}82.05 \mathrm{f} \\
\pm 0.022\end{array}$ & $\begin{array}{l}76.67 \mathrm{c} \\
\pm 0.154\end{array}$ & $\begin{array}{r}79.61 d \\
\pm 0.030\end{array}$ & $\begin{array}{l}81.72 \mathrm{e} \\
\pm 0.150\end{array}$ & $\begin{array}{l}75.83 b \\
\pm 0.011\end{array}$ & $\begin{array}{l}65.60 \mathrm{a} \\
\pm 0.086\end{array}$ \\
\hline Number of leaves & $\begin{array}{l}15.00 \mathrm{e} \\
\pm 0.00\end{array}$ & $\begin{array}{l}10.30 \mathrm{~b} \\
\pm 0.230\end{array}$ & $\begin{array}{l}10.23 \mathrm{~b} \\
\pm 0.020\end{array}$ & $\begin{array}{l}12.66 \mathrm{~d} \\
\pm 0.230\end{array}$ & $\begin{array}{l}11.00 \mathrm{c} \\
\pm 0.010\end{array}$ & $\begin{array}{c}9.00 \mathrm{a} \\
\pm 0.001\end{array}$ \\
\hline Leaf area $\left(\mathrm{cm}^{2}\right)$ & $\begin{array}{l}48.65 \mathrm{~d} \\
\pm 0.037\end{array}$ & $\begin{array}{l}40.38 \mathrm{c} \\
\pm 0.033 \\
\end{array}$ & $\begin{array}{l}46.00 \mathrm{~d} \\
\pm 0.040 \\
\end{array}$ & $\begin{array}{l}40.41 \mathrm{c} \\
\pm 0.004\end{array}$ & $\begin{array}{l}30.33 \mathrm{~b} \\
\pm 0.008\end{array}$ & $\begin{array}{l}22.16 \mathrm{a} \\
\pm 2.736\end{array}$ \\
\hline Root fresh weight (g) & $\begin{array}{c}0.88 \mathrm{~b} \\
\pm 0.004\end{array}$ & $\begin{array}{l}0.930 \mathrm{c} \\
\pm 0.004\end{array}$ & $\begin{array}{l}0.820 \mathrm{a} \\
\pm 0.004\end{array}$ & $\begin{array}{c}1.00 \mathrm{~d} \\
\pm 0.002\end{array}$ & $\begin{array}{l}0.950 \mathrm{e} \\
\pm 0.004\end{array}$ & $\begin{array}{l}0.810 \mathrm{a} \\
\pm 0.004\end{array}$ \\
\hline Shoot fresh weight (g) & $\begin{array}{c}1.72 \mathrm{~d} \\
\pm 0.013 \\
\end{array}$ & $\begin{array}{c}1.73 \mathrm{~d} \\
\pm 0.008 \\
\end{array}$ & $\begin{array}{c}1.44 \mathrm{c} \\
\pm 0.008 \\
\end{array}$ & $\begin{array}{r}1.41 \mathrm{c} \\
\pm 0.004 \\
\end{array}$ & $\begin{array}{r}1.28 \mathrm{~b} \\
\pm 0.020 \\
\end{array}$ & $\begin{array}{l}1.140 \mathrm{a} \\
\pm 0.008 \\
\end{array}$ \\
\hline Leaves fresh weight (g) & $\begin{array}{r}1.15 \mathrm{~d} \\
\pm 0.009 \\
\end{array}$ & $\begin{array}{c}0.970 \mathrm{ab} \\
\pm 0.212 \\
\end{array}$ & $\begin{array}{l}0.950 \text { bc } \\
\pm 0.008 \\
\end{array}$ & $\begin{array}{c}0.850 \mathrm{ab} \\
\pm 0.144 \\
\end{array}$ & $\begin{array}{c}0.720 \mathrm{ab} \\
\pm 0.004 \\
\end{array}$ & $\begin{array}{r}0.610 \mathrm{a} \\
\pm 0.008 \\
\end{array}$ \\
\hline Total plant fresh weight (g) & $\begin{array}{c}3.30 \mathrm{c} \\
\pm 0.115\end{array}$ & $\begin{array}{l}3.39 \text { bc } \\
\pm 0.206\end{array}$ & $\begin{array}{l}3.70 \text { bc } \\
\pm 0.122\end{array}$ & $\begin{array}{l}3.30 \text { bc } \\
\pm 0.017\end{array}$ & $\begin{array}{c}3.53 b \\
\pm 0.149\end{array}$ & $\begin{array}{c}2.96 \mathrm{a} \\
\pm 0.038\end{array}$ \\
\hline Root dry weight (g) & $\begin{array}{r}0.220 \mathrm{a} \\
\pm 0.400 \\
\end{array}$ & $\begin{array}{r}0.210 \mathrm{a} \\
\pm 0.004 \\
\end{array}$ & $\begin{array}{r}0.300 \mathrm{a} \\
\pm 0.005 \\
\end{array}$ & $\begin{array}{r}0.240 \mathrm{a} \\
\pm 0.008 \\
\end{array}$ & $\begin{array}{r}0.200 \mathrm{a} \\
\pm 0.004 \\
\end{array}$ & $\begin{array}{r}0.180 \mathrm{a} \\
\pm 0.010 \\
\end{array}$ \\
\hline Shoot dry weight (g) & $\begin{array}{l}0.210 \mathrm{~d} \\
\pm 0.012 \\
\end{array}$ & $\begin{array}{r}0.130 \mathrm{~b} \\
\pm 0.007\end{array}$ & $\begin{array}{l}0.200 \mathrm{~d} \\
\pm 0.018 \\
\end{array}$ & $\begin{array}{r}0.150 \mathrm{a} \\
\pm 0.015\end{array}$ & $\begin{array}{l}0.130 \mathrm{c} \\
\pm 0.008\end{array}$ & $\begin{array}{r}0.160 \mathrm{a} \\
\pm 0.004\end{array}$ \\
\hline Root / Shoot ratio & $\begin{array}{r}0.180 \mathrm{a} \\
\pm 0.005 \\
\end{array}$ & $\begin{array}{l}0.230 \mathrm{c} \\
\pm 0.001 \\
\end{array}$ & $\begin{array}{c}0.220 \text { bc } \\
\pm 0.003\end{array}$ & $\begin{array}{c}0.200 \mathrm{ab} \\
\pm 0.009\end{array}$ & $\begin{array}{l}0.300 \mathrm{~d} \\
\pm 0.010 \\
\end{array}$ & $\begin{array}{l}0.270 \mathrm{c} \\
\pm 0.008 \\
\end{array}$ \\
\hline Leaf weight ratio & $\begin{array}{l}0.850 \mathrm{~d} \\
\pm 0.004\end{array}$ & $\begin{array}{l}0.880 \mathrm{~d} \\
\pm 0.004\end{array}$ & $\begin{array}{l}0.740 \mathrm{c} \\
\pm 0.005\end{array}$ & $\begin{array}{l}0.610 \mathrm{~b} \\
\pm 0.012\end{array}$ & $\begin{array}{c}0.800 \mathrm{~d} \mathrm{~d} \\
\pm 0.034\end{array}$ & $\begin{array}{l}0.590 \mathrm{a} \\
\pm 0.007\end{array}$ \\
\hline Specific leaf area $\left(\mathrm{cm}^{2} \mathrm{~g}^{-1}\right)$ & $\begin{array}{l}0.280 \mathrm{~d} \\
\pm 0.002 \\
\end{array}$ & $\begin{array}{l}0.170 \mathrm{~b} \\
\pm 0.002 \\
\end{array}$ & $\begin{array}{l}0.250 \mathrm{c} \\
\pm 0.010 \\
\end{array}$ & $\begin{array}{l}0.180 \mathrm{~b} \\
\pm 0.004 \\
\end{array}$ & $\begin{array}{c}0.170 \mathrm{ab} \\
\pm 0.002 \\
\end{array}$ & $\begin{array}{r}0.170 \mathrm{a} \\
\pm 0.004 \\
\end{array}$ \\
\hline Leaf area ratio $\left(\mathrm{cm}^{2} \mathrm{~g}^{-1}\right)$ & $\begin{array}{l}2.180 \mathrm{c} \\
\pm 0.007\end{array}$ & $\begin{array}{c}2.30 \text { с } \\
\pm 0.244\end{array}$ & $\begin{array}{l}2.400 \mathrm{c} \\
\pm 0.020\end{array}$ & $\begin{array}{c}1.98 \mathrm{~b} \\
\pm 0.027\end{array}$ & $\begin{array}{c}1.94 \mathrm{~b} \\
\pm 0.023\end{array}$ & $\begin{array}{l}1.510 \mathrm{a} \\
\pm 0.012\end{array}$ \\
\hline
\end{tabular}

Table 4. Growth of mung bean in various concentrations of coriander powder extract.

\begin{tabular}{|l|c|c|c|c|c|c|}
\hline \multirow{2}{*}{$\begin{array}{l}\text { Seedling growth } \\
\text { characteristic }\end{array}$} & \multicolumn{5}{|c|}{ Treatments aqueous powder extract concentration (\%) } \\
\cline { 2 - 7 } & $\mathbf{0}$ & $\mathbf{1}$ & $\mathbf{2}$ & $\mathbf{3}$ & $\mathbf{4}$ & $\mathbf{5}$ \\
\hline \multirow{2}{*}{ Shoot length (cm) } & $48.21 \mathrm{e}$ & $45.31 \mathrm{~b}$ & $45.42 \mathrm{c}$ & $46.13 \mathrm{~d}$ & $46.22 \mathrm{~d}$ & $40.23 \mathrm{a}$ \\
& \pm 0.008 & \pm 0.004 & \pm 0.029 & \pm 0.010 & \pm 0.027 & \pm 0.016 \\
\hline \multirow{2}{*}{ Root length (cm) } & $12.43 \mathrm{~d}$ & $13.37 \mathrm{de}$ & $11.90 \mathrm{a}$ & $12.30 \mathrm{c}$ & $12.33 \mathrm{c}$ & $6.90 \mathrm{~b}$ \\
& \pm 0.020 & \pm 0.010 & \pm 0.040 & \pm 0.062 & \pm 0.009 & \pm 0.016 \\
\hline \multirow{2}{*}{ Seedling length (cm) } & $60.64 \mathrm{e}$ & $56.68 \mathrm{~d}$ & $57.32 \mathrm{~b}$ & $58.43 \mathrm{c}$ & $58.55 \mathrm{c}$ & $47.13 \mathrm{a}$ \\
& \pm 0.014 & \pm 0.014 & \pm 0.056 & \pm 0.057 & \pm 0.030 & \pm 0.029 \\
\hline \multirow{2}{*}{ Number of leaves } & $10.00 \mathrm{~b}$ & $8.35 \mathrm{a}$ & $9.00 \mathrm{~b}$ & $10.12 \mathrm{~b}$ & $9.66 \mathrm{~b}$ & $7.22 \mathrm{~b}$ \\
& \pm 0.00 & \pm 0.781 & \pm 0.00 & \pm 0.409 & \pm 0.034 & \pm 0.001 \\
\hline \multirow{2}{*}{ Leaf area $\left(\mathrm{cm}^{2}\right)$} & $22.80 \mathrm{a}$ & $22.97 \mathrm{ab}$ & $23.80 \mathrm{~b}$ & $25.00 \mathrm{c}$ & $22.90 \mathrm{a}$ & $17.00 \mathrm{c}$ \\
& \pm 0.080 & \pm 0.040 & \pm 0.080 & \pm 0.062 & \pm 0.022 & \pm 0.81 \\
\hline
\end{tabular}


ALLELOPATHIC POTENTIAL OF CAPSICUM ANNUUM L. AND CORIANDRUM SATIVUM L. ON...

\begin{tabular}{|c|c|c|c|c|c|c|}
\hline Root fresh weight (g) & $\begin{array}{l}0.390 \mathrm{a} \\
\pm 0.007\end{array}$ & $\begin{array}{l}0.650 \mathrm{~b} \\
\pm 0.010\end{array}$ & $\begin{array}{l}0.980 \mathrm{~d} \\
\pm 0.047\end{array}$ & $\begin{array}{l}0.610 \mathrm{~b} \\
\pm 0.004\end{array}$ & $\begin{array}{l}0.780 \mathrm{c} \\
\pm 0.006\end{array}$ & $\begin{array}{l}0.090 \mathrm{~b} \\
\pm 0.004\end{array}$ \\
\hline Shoot fresh weight (g) & $\begin{array}{l}1.630 \mathrm{c} \\
\pm 0.004 \\
\end{array}$ & $\begin{array}{l}1.650 \mathrm{c} \\
\pm 0.004 \\
\end{array}$ & $\begin{array}{l}1.770 \mathrm{~d} \\
\pm 0.017 \\
\end{array}$ & $\begin{array}{r}1.720 \mathrm{e} \\
\pm 0.006 \\
\end{array}$ & $\begin{array}{l}1.540 \mathrm{~b} \\
\pm 0.004 \\
\end{array}$ & $\begin{array}{l}1.010 \mathrm{a} \\
\pm 0.011 \\
\end{array}$ \\
\hline Leaves fresh weight (g) & $\begin{array}{l}1.280 \mathrm{f} \\
\pm 0.004\end{array}$ & $\begin{array}{l}1.090 \mathrm{~d} \\
\pm 0.010\end{array}$ & $\begin{array}{l}1.090 \mathrm{~d} \\
\pm 0.010\end{array}$ & $\begin{array}{l}0.950 \mathrm{a} \\
\pm 0.019\end{array}$ & $\begin{array}{l}1.210 \mathrm{e} \\
\pm 0.004\end{array}$ & $\begin{array}{l}0.620 \mathrm{c} \\
\pm 0.006\end{array}$ \\
\hline Total plant fresh weight (g) & $\begin{array}{c}3.30 \mathrm{~b} \\
\pm 0.125\end{array}$ & $\begin{array}{l}3.390 \mathrm{c} \\
\pm 0.017\end{array}$ & $\begin{array}{l}3.700 \mathrm{e} \\
\pm 0.048\end{array}$ & $\begin{array}{l}3.300 \mathrm{~b} \\
\pm 0.008\end{array}$ & $\begin{array}{l}3.530 \mathrm{~d} \\
\pm 0.004\end{array}$ & $\begin{array}{l}1.720 \mathrm{a} \\
\pm 0.006\end{array}$ \\
\hline Root dry weight (g) & $\begin{array}{l}0.170 \mathrm{~b} \\
\pm 0.005\end{array}$ & $\begin{array}{l}0.250 \mathrm{~d} \\
\pm 0.004\end{array}$ & $\begin{array}{l}0.480 \mathrm{e} \\
\pm 0.008\end{array}$ & $\begin{array}{l}0.210 \mathrm{c} \\
\pm 0.004\end{array}$ & $\begin{array}{l}0.190 \mathrm{~b} \\
\pm 0.004\end{array}$ & $\begin{array}{l}0.070 \mathrm{a} \\
\pm 0.008\end{array}$ \\
\hline Shoot dry weight (g) & $\begin{array}{l}1.030 \mathrm{~b} \\
\pm 0.004\end{array}$ & $\begin{array}{l}1.250 \mathrm{~d} \\
\pm 0.004\end{array}$ & $\begin{array}{l}1.370 \mathrm{f} \\
\pm 0.004\end{array}$ & $\begin{array}{l}1.300 \mathrm{e} \\
\pm 0.004\end{array}$ & $\begin{array}{l}1.140 \mathrm{c} \\
\pm 0.004\end{array}$ & $\begin{array}{l}0.910 \mathrm{a} \\
\pm 0.004\end{array}$ \\
\hline Leaves dry weight (g) & $\begin{array}{r}0.980 \mathrm{a} \\
\pm 0.217\end{array}$ & $\begin{array}{r}0.590 \mathrm{a} \\
\pm 0.008\end{array}$ & $\begin{array}{l}0.550 \mathrm{a} \\
\pm 0.097\end{array}$ & $\begin{array}{l}0.470 \mathrm{a} \\
\pm 0.004\end{array}$ & $\begin{array}{l}0.610 \mathrm{a} \\
\pm 0.005\end{array}$ & $\begin{array}{c}0.530 \mathrm{ab} \\
\pm 0.019\end{array}$ \\
\hline Total plant dry weight (g) & $\begin{array}{l}2.180 \mathrm{~b} \\
\pm 0.210\end{array}$ & $\begin{array}{l}2.090 \mathrm{~b} \\
\pm 0.010\end{array}$ & $\begin{array}{l}2.400 \mathrm{c} \\
\pm 0.012\end{array}$ & $\begin{array}{l}1.980 \mathrm{~b} \\
\pm 0.010\end{array}$ & $\begin{array}{l}1.940 \mathrm{~b} \\
\pm 0.008\end{array}$ & $\begin{array}{l}1.510 \mathrm{a} \\
\pm 0.021\end{array}$ \\
\hline Leaf weight ratio & $\begin{array}{r}0.450 \mathrm{e} \\
\pm 0.007 \\
\end{array}$ & $\begin{array}{l}0.280 \mathrm{c} \\
\pm 0.150 \\
\end{array}$ & $\begin{array}{l}0.220 \mathrm{c} \\
\pm 0.004 \\
\end{array}$ & $\begin{array}{l}0.230 \mathrm{~b} \\
\pm 0.006 \\
\end{array}$ & $\begin{array}{l}0.310 \mathrm{~d} \\
\pm 0.001 \\
\end{array}$ & $\begin{array}{l}0.340 \mathrm{~d} \\
\pm 0.005 \\
\end{array}$ \\
\hline Specific leaf area $\left(\mathrm{cm}^{2} \mathrm{~g}^{-1}\right)$ & $\begin{array}{c}23.260 \mathrm{a} \\
\pm 0.094\end{array}$ & $\begin{array}{c}38.390 \mathrm{c} \\
\pm 0.123\end{array}$ & $\begin{array}{c}43.270 \mathrm{~d} \\
\pm 0.146\end{array}$ & $\begin{array}{c}53.190 \mathrm{f} \\
\pm 0.159\end{array}$ & $\begin{array}{c}37.540 \mathrm{~b} \\
\pm 0.104\end{array}$ & $\begin{array}{c}47.160 \mathrm{e} \\
\pm 0.242\end{array}$ \\
\hline Leaf area ratio $\left(\mathrm{cm}^{2} \mathrm{~g}^{-1}\right)$ & $\begin{array}{c}10.450 \mathrm{~b} \\
\pm 0.107\end{array}$ & $\begin{array}{c}10.990 \mathrm{c} \\
\pm 0.033\end{array}$ & $\begin{array}{l}9.910 \mathrm{a} \\
\pm 0.042\end{array}$ & $\begin{array}{c}12.620 \mathrm{~d} \\
\pm 0.036\end{array}$ & $\begin{array}{c}11.800 \mathrm{~d} \\
\pm 0.067\end{array}$ & $\begin{array}{c}16.550 \mathrm{e} \\
\pm 0.103\end{array}$ \\
\hline
\end{tabular}

The seedlings of cowpea were tested to the tolerance to different concentrations of red chilli and coriander powder extract. Red chili extract treatment at 1, 2, 3, 4 and 5\% gradually decreased tolerance indices by $94.50,84.70,77.41,74.50$ and $65.79 \%$ in seedlings of cowpea. The treatment of coriander extract treatment at $5 \%$ showed the lowest tolerance (67.45\%) indices. However, on comparison, the seedlings of cowpea showed more tolerance to coriander powder extract than red chili extract at 5\%. Red chilli extract treatment at 4 and $5 \%$ showed tolerance indices by 64.84 and $55.51 \%$ in seedlings of mung bean. Coriander extract treatment at all concentrations showed better tolerance in seedlings of mung bean as compared to red chilli extract at similar concentrations.

\section{Discussion}

The studies on interactions and competition for resources among plants was carried out and suggested for the use in modifying the pattern of crop cultivation and for the increase in yields [MAJEED \& al. 2017]. The treatment of red chili and coriander aqueous powder extract influenced on seedling growth performances of cowpea and mung bean. The agrochemical groups are naturally occurred in plant [KUTI \& al. 1990] and produced favorable and toxic impact on the growth of surrounding plants. It was found that the release of chemical compounds from both home spices in the given substrate affected growth performance of both beans. The different response in seedling growth parameters of bean seems attributable to the level of red chili and coriander powder extract treatments. Allelopathic compounds from plants residues called allelochemicals and may work as inhibitor or beneficial for growth of subsequent plants [RICE, 1984]. The maximum 
inhibitory allelopathic effect of bindweed (Convolvulus arvensis L.) vegetative part at 10\% on seed vigor index for millet and basil plants was observed [FATEH \& al. 2012]. The inhibitory substances from C. annuum and C. sativum released in the soil which apparently showed allelopathic potential and might be cause for significant decline in growth characteristics of cowpea and agreed with the findings of ELLS \& MCSAY (1991) on cucumber seedlings by alfalfa plant residues. Capsaicin have a powerful allelopathic effect on growth of plant [CHO \& al. 1992] and growth of weed [GONZALEZ \& al. 1997]. The treatment of both spices at $2 \%$ level further decreased root and shoot growth of cowpea. MOOSAVI \& al. (2011) also found significantly decreased in shoot and root growth of Vigna radiata $\mathrm{L}$. with aqueous extract of leaf, stem and root of sorghum. The treatment of ccoriander powder extract showed beneficial and harmful effects on the seedling characteristics of cowpea. The low concentration of coriander powder at $1 \%$ slightly increased and a higher concentration decreased the seedling dry weight of cowpea. The presence of phenols and tannins from Jatropha curcas (5-20\%) as allelochemicals showed inhibitory effect on green chilli and stimulatory on sesame [REJILA \& VIJAYAKUMAR, 2011].

\section{Conclusions}

It was concluded that aqueous powder extract of red chili and coriander at 5\% influenced on seedling growth and tolerance index of cowpea and mung bean. The availability of toxic allelochemicals compounds from both spices in substrate showed strong allelopathic potential activity for seedling of cowpea and mung bean.

\section{Notes on contributors}

Prof. Dr. Muhammad Zafar IQBAL, PhD, is ex-chairman department of Botany, University of Karahi, and has special interest in plant ecology and pollution, climatology, plant and environment, automobile pollution, industrial pollution and ecology and has published more than 170 scientific research papers in national and international scientific peer reviewed renowned scientific journals.

Lubna AHMED is MS student in Botany.

Dr. Muhammad SHAFIQ, PhD, is a research scholar and have main interest in plant ecology and environmental pollution, phytosociology. He has published more than 110 scientific research papers in national and international scientific peer reviewed renowned scientific journals. He has also published books on "Impact of Automobile Pollutants on Plants" and "Poison Land. Vegetation of disturbed and polluted areas in Pakistan”. He has also published few book chapters.

\section{Acknowledgements}

The authors sincerely acknowledged the experimental facilities provided by the Chairman, Department of Botany, University of Karachi.

\section{References}

ABBASSI F., GHORBANI R. \& KHORRAMDEL S. 2013. Allelopathy research in Iran: experiences, challenges, and prospects: 159-192. In: CHEEMA Z., FAROOQ M. \& WAHID A. (eds.). Allelopathy. Springer, Berlin, Heidelberg. https://doi.org/10.1007/978-3-642-30595-5_8

ALLOLI T. B. \& NARAYANREDDY P. 2000. Allelopathic effects of Eucalyptus plant extracts on germination and seedling growth of cucumber. Karnataka Journal of Agricultural Sciences. 13(4): 947-951.

BAGHERI A., REZAEI M. \& EIVAZI A. R. 2013. Allelopathic properties of Chenopodium album on seedling related traits of sorghum cultivars. International Journal of Agronomy and Plant Production. 4(S): 3743-3747.

BOJOVIĆ B. M. \& JAKOVLJEVIĆ D. Z. 2015. Allelopathic relations of selected cereal and vegetable species during seed germination and seedling growth. Kragujevac Journal of Sciences. 37: 135-142. https://doi.org/10.5937.//KGJSC11537135B 
CHEEMA Z. A., FAROOQ M. \& KHALIQ A. 2013. Application of allelopathy in crop production: success story from Pakistan: 113-143. In: CHEEMA Z., FAROOQ M. \& WAHID A. (eds.) Allelopathy. Springer, Berlin, Heidelberg. https://doi.org/10.1007/978-3-642-30595-5_6

CHO K. J., PARK K. S. \& TANG C. S. 1992. Allelopathic potential of red-pepper (Capsicum annuum L.) fruit. Research Report to Rural Development Administration. 34: 18-23.

CHOU C. H. \& LEE Y. F. 1991. Allelopathic dominance of Miscanthus transmorrisonensis in an alpine grassland community in Taiwan Journal of Chemical Ecology. 17(11): 2267-2281. https://doi.org/10.1007/ BF00988007

DAIZY R., BATISH H. P. \& SINGH S. K. 2001. Crop allelopathy and its role in ecological agriculture. Journal of Crop Production. 4(2): 121-161.

DONGRE P. N. \& YADAV B. 2005. Inhibitory allelopathic effect of weed leaf leachates on seed germination of pea (Pisum sativum L.). Crop Research Hisar. 29: 458-461.

ELLS J. E. \& MCSAY A. E. 1991. Allelopathic effects of alfalfa plant residues on emergence and growth of cucumber seedlings. Horticultural Science. 26(4): 368-370.

FATEH E., SOHRABI S. S. \& GERAMI F. 2012. Evaluation the allelopathic effect of bindweed (Convolvulus arvensis L.) on germination and seedling growth of millet and basil. Advances in Environmental Biology. 6(3): 940-950.

FERGUSON J. J. \& RATHINASABPATHI B. 2003. Allelopathy: How plants suppress other plants. Horticultural Sciences Department, Florida Cooperative Extension Service. pp. 1-5. http://edis.ifas.ufl.edu.

GONZALEZ L., SOUTO X. C. \& REIGOSA M. J. 1997. Weed control by Capsicum annuum. Allelophaty Journal. 4: 101-110.

HEGAZY A. K. L., MANSOUR K. S. \& ABDEL-HADY N. F. 1990. Allelopathic and autotoxic effects of Anastatica hierochuntica. Journal of Chemical Ecology. 16(7): 2183-2193.

KIL B. S. \& YUN K. W. 1992. Allelopathic effects of water extracts of Artemisia princeps var. orientalis on selected plant species. Journal of Chemical Ecology. 18(1): 39-51.

KUTI J. O., JARVIS B. B., MOKHTARI-REJALI N. \& BEAN G. A. 1990. Allelochemical regulation of reproduction and seed germination of two Brazilian Baccharis species by phytotoxic trichothecenes. Journal of Chemical Ecology. 16 (12): 3441-3453.

MAJEED A., MUHAMMAD Z., HUSSAIN M. \& AHMAD H. 2017. In vitro, allelopathic effect of aqueous extracts of sugarcane on germination parameters of wheat. Acta Agriculturae Slovenica. 109(2): 349-356.

MELKANIA N. P. 1984. Influence of leaf leachets of certain woody spp. on agriculture crops. Indian Journal of Ecology. 11: 82-86.

MISHRA A. 2014. Phytotoxic effect of Lantana camara leaf extract on germination and growth of Pisum sativum. Indian Journal of Plant Research. 4(5): 55-56.

MOOSAVI A., TAVAKKOL R., AFSHARI A., ASADI A. \& GHARINEH M. S. 2011. Allelopathic effects of aqueous extract of leaf stem and root of sorghum bicolor on seed germination and seedling growth of Vigna radiata L. Notulae Scientia Biologicae. 3(2): 114-118.

OUSSAMA O. 2003. Allelopathy in two durum wheat (Triticum durum L.) varieties. Agriculture Ecosystems \& Environment. 96: 161-163.

PURI S. \& KHARA A. 1991. Allelopathic effects of Eucalyptus tereticornis on Phaseolus vulgaris seedlings. The International Tree Crops Journal. 6(4): 287-293.

REHMAN S. A. \& IQBAL M. Z. 2009. Growth of Leucaena leucocephala (Lam.) De-wit in different soil compositions of Korangi and Landhi industrial areas of Karachi, Pakistan. Pakistan Journal of Botany. 41: 3125-3138.

REINHARDT C. F., MEISSNER R. \& NEL P. C. 1993. Allelopathic effect of sweet potato (Ipomoea batatas) cultivars on certain weed and vegetable species. South African Journal of Plant and Soil. 10(1): 41-44.

REJILA S. \& VIJAYAKUMAR N. 2011. Allelopathic effect of Jatropha curcas on selected intercropping plants (Green Chilli and Sesame). Journal of Phytopharmacology. 3(5): 1-3.

RICE E. L. 1984. Allelopathy. $2^{\text {nd }}$ ed., Academic Press, New York: 421 pp.

ZACKRISSON O. \& NILSSON M. C. 1992. Allelopathic effects by Empetrum hermaphroditum on seed germination of two boreal tree species. Canadian Journal of Forest Research. 22(9): 1310-1319.

How to cite this article:

IQBAL M. Z., AHMED L. \& SHAFIQ M. 2020. Allelopathic potential of Capsicum annuum L. and Coriandrum sativum L. on growth of bean crop. J. Plant Develop. 27: 63-70. https://doi.org/10.33628/jpd. 2020.27.1.63 